Ocula ${ }^{15}$

Commemorating Charles S. Peirce (1839-1914):

interpretive semiotics and mass media

A cura di Giampaolo Proni e Salvatore Zingale

Novembre 2014 I www.ocula.it - DOI: 10.12977/ocula30

\title{
Consumo di merci-segni e di segni-merci nella riproduzione sociale
}

\author{
Giorgio Borrelli \\ Università di Bari \\ giorgioborrelli83@yahoo.it
}

\begin{abstract}
Prendendo le mosse dal materialismo pragmaticista di Bonfantini e dal metodo omologico di Rossi-Landi, questo articolo propone dei lineamenti teorici per un'analisi critica della categoria di consumo.

Secondo Rossi-Landi, il momento economico del consumo - esattamente come i momenti della produzione e dello scambio - si articola attraverso determinati programmi semiotici e non può prescindere da essi. Dunque, porre in essere determinati comportamenti di consumo corrisponde - nella prospettiva materialistico-dialettica di Rossi-Landi - ad erogare forza lavoro linguistica; consumare rispettando certi codici significa eseguire determinati programmi stabiliti dalla logica della produzione capitalistica.

In sostanza, i programmi di consumo - in quanto abiti - richiedono interpretazione e dispongono ad agire. Ma tali programmi possono anche essere messi in discussione e risemantizzati attraverso un lavoro linguistico emancipante, dando così origine ad una prassi trasformativa. Questa dinamica può essere letta alla luce della logica elementare del processo di azione - strutturata da Bonfantini nella sua rielaborazione materialistica dell'abduzione peirceana. Più precisamente, propongo di analizzare la categoria di consumo - nella sua accezione di comportamento semioticamente programmato attraverso la cadenza di rappresentazione, proiezione e programmazione.

In sintesi, una semiotica orientata in senso materialista e pragmaticista può costituire uno strumento critico per l'analisi dei comportamenti di consumo nel processo di riproduzione sociale.
\end{abstract}

\section{Parole chiave}

Artefatto, Consumo, Materialismo dialettico, Pragmaticismo, Programma

\section{Sommario}

1. Introduzione. La dialettica produzione-consumo come Secondità, Primità e Terzità

2. Programmi, comportamenti e artefatti

3. La semiosi dell'invenzione nella produzione e nel consumo

4. Conclusione. Verso una semiotica dei programmi di scambio

Bibliografia 


\section{Ocula $a^{15}$}

Commemorating Charles S. Peirce (1839-1914): interpretive semiotics and mass media

Giorgio Borrelli, Consumo di merci-segni e di segni-merci nella riproduzione sociale

\section{Introduzione. La dialettica produzione-consumo come Secon- dità, Primità e Terzità}

Attraverso il concetto di riproduzione sociale, Ferruccio Rossi-Landi (1921-1985) intende descrivere «l'insieme dei processi per mezzo dei quali una comunità o società sopravvive, accrescendosi o almeno continuando ad esistere» (Rossi-Landi [1985] 2006: 175). Prendendo le mosse dal Marx dei Grundrisse - e in particolare dalla loro "Einleitung" ("Introduzione") del 1857 (Marx 1968: 1-40 tr. it.) -, Rossi-Landi sostiene che in ciascun processo di riproduzione sociale si possano individuare tre momenti economici: produzione, scambio e consumo.

Questi tre momenti sono irriducibili, nel senso che senza di essi non si ha riproduzione umana [...]. Produzione, scambio e consumo sono anzi così strettamente interconnessi che se ne può parlare separatamente solo per deliberata astrazione. I beni di cui gli umani hanno bisogno, i "valori d'uso", debbono infatti essere prodotti e consumati, consumati e prodotti; con la divisione sociale del lavoro, fra i due momenti astrattamente iniziale e terminale della produzione si inserisce quale momento astrattamente intermedio lo scambio dei beni prodotti. (Rossi-Landi [1985] 2006: 28)

L'irriducibilità di produzione, scambio e consumo dipende dal fatto che essi, pur essendo «inestricabilmente connessi» (ivi: 180), in quanto appartenenti «alla stessa totalità» (ibidem, corsivo mio) - costituita, appunto, dalla riproduzione sociale -, non sono momenti identici; dunque, «la loro unità è dialettica» (ibidem).

A mio modo di vedere, un simile rapporto di distinzione nell'ambito di un'unità - per continuare ad usare un linguaggio marxiano (v. Marx 1968: 25 tr. it.) - è ritrovabile nel modo in cui Peirce struttura la sua dottrina delle categorie. Difatti, fa notare Bonfantini,

Le tre categorie sono, secondo Peirce, sempre $a$ ) compresenti, $b$ ) interdipendenti, c) irriducibili l'una all'altra.

Ciò significa che - in ogni momento d'esperienza, in ogni atto di cognizione, in ogni significazione - tutte e tre le categorie si dànno solidalmente, ognuna svolgendo una sua funzione specifica, e ognuna provocando l'occorrere delle altre due secondo l'effettualità genetica che le è propria. (Bonfantini 2003: 27-28)

Dunque, possiamo dire che produzione, scambio e consumo - esattamente come Primità, Secondità e Terzità - siano sempre in rapporto dialettico come momenti compresenti, interdipendenti, eppure reciprocamente irriducibili. Inoltre, produzione, scambio e consumo, sono compresenti in ogni processo della riproduzione sociale, così come le categorie di Primità, Secondità e Terzità sono presenti nella continuità del faneron, cioè in «tutto ciò che appare» (Proni 1990: 184). In base al principio di compresenza, o sinechismo, nel faneron «le categorie non sono mai isolabili in maniera assoluta» (ivi).

A partire da queste considerazioni preliminari, si potrebbe immaginare una suggestiva - a mio parere - ipotesi di ricerca, volta ad individuare una 


\section{Ocula $a^{15}$}

Commemorating Charles S. Peirce (1839-1914): interpretive semiotics and mass media

Giorgio Borrelli, Consumo di merci-segni e di segni-merci nella riproduzione sociale

comune radice tra le due triadi. Forse, questa comune radice potrebbe essere hegeliana; del resto, è proprio Peirce ad ammettere che le sue categorie «si accordano sostanzialmente [...] con quelle di Hegel» (CP 2.87 2003: 122); così come, è proprio Marx nell'“Einleitung” a prendere le distanze da Hegel e dalla sua «illusione di concepire il reale come risultato del pensiero» (ivi: 27). Un'interpretazione critica di Hegel su cui probabilmente Peirce sarebbe stato d'accordo, ma che secondo alcuni (v. Stern 2009) non corrisponderebbe a ciò che Hegel intendeva... realmente!

A prescindere dalla ricerca della radice hegeliana, potremmo spingere oltre la nostra ipotesi, cercando di analizzare le varie modalità del «movimento di mediazione» (Marx 1968: tr. it. 15) tra produzione e consumo alla luce delle categorie peirceane di Orienza (Primità, o Originarietà), Obsistenza (o Secondità, o Binarità) e Transuasione (o Terzità, o Mediazione). Per essere precisi, dovremmo rispettare «l'ordine in cui le categorie emergono nell'esperienza, [e cioè] Secondità, Primità, Terzità» (Proni 1990: 194).

Partendo dunque dalla Secondità, e provando ad includere nella nostra analisi la teoria della degenerazione delle categorie (cfr. ivi: 207-210), potremmo definire la dialettica tra produzione e consumo come una relazione reale, cioè una relazione «che sussiste in virtù di un fatto che sarebbe totalmente impossibile se uno qualunque degli oggetti relati fosse distrutto» (ivi: 208). Allo stesso modo - logicamente! - non sussisterebbe alcuna relazione tra produzione e consumo se venisse a mancare uno di questi due elementi o, per dirla proprio con Marx, «senza produzione, niente consumo; senza consumo, niente produzione» (Marx 1968: tr. it. 17).

Più complesso - se non impossibile - sarebbe considerare queste due categorie nella loro Originarietà; sarebbe molto difficile, ad esempio, analizzare la produzione come Possibilità, come «mera potenzialità astratta» (CP. 1422, tr. it. Proni 1990: 197). Per di più, se per Peirce è errato ridurre il potenziale, il possibile, a «ciò che l'effettivo [actual] lo fa essere» (CP. 1422, tr. it. Proni 1990: 197), per Marx è invece vero il contrario: la produzione senza l'effettua-

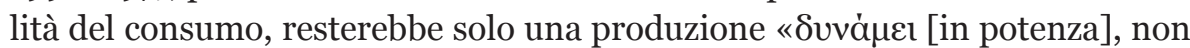
in realtà» (Marx 1968: tr. it. 15). È l'effettività del consumo che rende tale la produzione e allo stesso modo è la produzione che rende effettivo il consumo, determinandone le modalità. Ciascuno dei due momenti economici «realizzandosi crea l'altro, si realizza come l'altro» (ivi: 17).

Forse, proprio la categoria di Mediazione potrebbe essere quella più adatta per una lettura "peirceana"1 del movimento - appunto - di mediazione tra produzione e consumo. In particolare, dovremmo considerare la Terzità nella sua dimensione temporale, come essere in futuro. Secondo Peirce, «il futuro ha effetto sul presente, sul flusso della conoscenza, in quanto fine o scopo di un'azione» (Proni 1990: 204). Il futuro - a differenza del passato - influisce sul presente in maniera indiretta, tramite l'azione mediatrice dell'intenzione, che si configura come «ragionamento sull'assente possibile» (ibidem). L'as-

1 Utilizzerò d'ora in poi "peirceano" - tra virgolette - per riferirmi alle interpretazioni e agli approcci teorici che fanno riferimento o rielaborano la semiotica di Peirce. Utilizzerò lo stesso termine fuori dalle virgolette quando vorrò riferirmi direttamente al pensiero di Peirce. 


\section{Ocula $a^{15}$}

Commemorating Charles S. Peirce (1839-1914): interpretive semiotics and mass media

Giorgio Borrelli, Consumo di merci-segni e di segni-merci nella riproduzione sociale

sente possibile «è l'oggetto del desiderio che, insoddisfatto, viene proiettato e interpretato in altri segni, diventa l'obiettivo di un pensiero che può produrre l'azione per congiungersi con esso» (Proni 2012: 100). In questa prospettiva, l'assente possibile diviene uno dei due elementi alla base della semiosi dell'invenzione progettuale (v. Bonfantini 2004): il progetto è l'interpretazione di un desiderio, inteso come ragionamento sull'assente possibile. Nelle pagine seguenti cercherò di mostrare come la dialettica tra produzione e consumo si articoli in base ai modelli semiotici - o progetti - che ciascuno dei due momenti economici struttura per soddisfare i propri desideri, intesi come interpretazioni dei propri bisogni.

L'artefatto, o oggetto d'uso, costituirà il punto di partenza di questa analisi, perché nell'artefatto convergono i progetti della produzione e a partire dall'artefatto si irradiano - sotto forma di uso - i progetti del consumo. Ritengo che un'analisi di questo genere possa essere condotta facendo incontrare prospettive teoriche diverse. Nello specifico, la semiotica degli artefatti che Proni (2012: 38-70) delinea a partire dalla definizione peirceana di "Segno" può fungere da "cerniera" tra una semiotica orientata in senso materialistico-dialettico (v. Ponzio 2004; Petrilli 2004; Rossi-Landi [1985] 2006) e una semiotica materialistico-pragmaticista (Bonfantini 1987; 2013), ${ }^{2}$ creando tra questi due approcci nuove occasioni di dialogo e delineando una comune prospettiva teorico-critica (e dunque, anche politica): un'analisi critica degli oggetti d'uso - e dei comportamenti di consumo che ne costituiscono gli interpretanti - implica necessariamente un'analisi critica del sistema economico che quegli oggetti produce. La critica del consumo è anche critica della produzione.

\section{Programmi, comportamenti e artefatti}

Prendendo le mosse dalla celebre definizione peirceana del segno come «qualcosa che sta a qualcuno, per qualcosa, sotto qualche rispetto o capacità» (CP 2.228 2003: 147), Proni sostiene che anche gli oggetti d'uso possano essere analizzati semioticamente. Nello specifico, l'analisi semiotica - a partire dalla triade Segno, Oggetto, Interpretante - può mettere in evidenza delle caratteristiche distintive degli oggetti d'uso (o artefatti): «1. La loro qualità materiale o rapresentamen è normalmente usata per scopi pratici, in interazione con altre entità fisiche, incluso il corpo umano; 2. Il loro Oggetto descrive come usare la qualità materiale per ottenere determinati risultati generali; 3. Il loro Interpretante 'standard' descrive i risultati specifici che si vogliono ottenere» (Proni 2012: 48).

In questa prospettiva, l'uso di un artefatto viene a configurarsi come un tipo particolare di semiosi e "gli oggetti d'uso sono i segni che la rendono possibile» (ivi: 47); «l'Oggetto di un artefatto è una funzione, e punta alla parte materiale del Segno come mezzo per svolgerla» (ivi: 48); più precisa-

2 Un simile incontro di prospettive non è poi così difficile da immaginare qualora si consideri il metodo omologico di Rossi-Landi come un metodo abduttivo (v. Ponzio 2012: 112-116). 
Giorgio Borrelli, Consumo di merci-segni e di segni-merci nella riproduzione sociale

mente, la funzione principale di ogni artefatto è una funzione pratica, ossia, una funzione legata all'ottenimento di determinati risultati: un oggetto d'uso deve funzionare. Infine, l'Interpretante è il tipo di risposta che la funzione genera nell'interprete. Tale risposta, trattandosi dell'uso di un artefatto, deve configurarsi come un «comportamento adeguato» (ivi:41). In sostanza, l'utente-interprete deve imparare a svolgere correttamente la funzione pratica dell'artefatto, deve imparare ad usare l'artefatto correttamente.

A mio modo di vedere, un primo elemento di convergenza tra l'approccio "peirceano" e quello rossilandiano potrebbe essere rinvenuto proprio in questa relazione tra le funzioni degli oggetti ed i comportamenti che le interpretano. Secondo Rossi-Landi, «usare una cosa significa comportarsi con essa, o rispetto a essa» (Rossi-Landi [1985] 2006: 161). Dunque, il comportamento dell'utente - il modo in cui questi usa-consuma l'oggetto - dipenderebbe dall'interpretazione del particolare programma d'uso inscritto nella cosa, nell'oggetto d'uso. ${ }^{3}$

Un altro elemento di convergenza - strettamente collegato al primo - potrebbe essere la visione processuale dell'artefatto. L'Oggetto di un artefatto, sottolinea Proni, possiede un doppio carattere:

da una parte l'Oggetto è il processo di produzione, che rappresenta il passato dell'artefatto, la catena di cause ed effetti che lo ha prodotto come entità materiale; dall'altra l'Oggetto è l'interazione possibile, la funzione, cioè il suo futuro [...]. L'Oggetto, inoltre, rinvia all'indietro alla qualità materiale del Segno come mezzo per mettere in atto la funzione.

Questo significato è acquisito e attualizzato solo nell'Interpretante, ma punta sempre a una parte $o$ aspetto del Segno. L'interpretante può attivare uno solo o entrambi gli aspetti dell'Oggetto. (ivi: 52)

Dunque, il processo di produzione e la funzione devono essere analizzati come momenti discreti di uno stesso continuum semiotico, di cui il corpo dell'artefatto è la parte materiale. Più specificamente,

Uso e produzione sono le due direzioni della linea causale sulla quale l'oggetto d'uso è situato come evento materiale. La produzione è la catena di cause ed effetti che ha portato l'oggetto d'uso a esistere. L'uso è la catena di cause ed effetti possibili e probabili in cui l'oggetto d'uso sarà coinvolto. L'oggetto è di fatto un processo, che si interseca con il processo d'uso dell'utente. In questo processo sono inscritte le descrizioni di due soggetti: l'utente modello e il produttore implicito. (ivi: 56)

3 Non mi sembra errato affermare che l'analisi del concetto di "comportamento" sia stata una delle costanti del lavoro di ricerca di Rossi-Landi. Non posso ricostruire qui le varie articolazioni di questa analisi. Per un approfondimento rimando direttamente a Ponzio (2012) e Rossi-Landi ([1972] 2011: 196-209; 1975; [1978] 2005: 141-166; [1985] 2006: 107-110). Per un confronto tra Rossi-Landi e la semiotica comportamentistica di Morris v. Petrilli (1999: 9-52).

4 L'utente modello è l'«enunciatario della produzione» (ivi: 59), cioè l'utente chiamato ad interpretare la funzione che il produttore implicito (enuciatore) ha inscritto nell'artefatto. 


\section{Ocula $a^{15}$}

Commemorating Charles S. Peirce (1839-1914): interpretive semiotics and mass media

Giorgio Borrelli, Consumo di merci-segni e di segni-merci nella riproduzione sociale

A mio modo di vedere, Rossi-Landi imposta la sua analisi degli oggetti d'uso in modo non dissimile, cercando di mostrare come le dimensioni del lavoro produttivo e dell'uso-consumo possano sovrapporsi e contemporaneamente differenziarsi; in ogni caso, solo partendo dall'artefatto si può distinguere la produzione dall'uso, oppure evidenziare la loro continuità e reciproca interazione; questo perché nell'artefatto converge «una tipica situazione dialettica di unità-distinzione. L'uso è lavoro; al tempo stesso, l'uso si distingue dal lavoro» (Rossi-Landi [1985] 2006: 19). Nello specifico, l'uso coincide con il lavoro perché l'uso di ogni artefatto avviene necessariamente in base ad «operazioni programmate» (ibidem). In questa prospettiva - sostiene Rossi-Landi - l'uso-consumo rientra nell'accezione marxiana del lavoro come zweckmässige Tätigkeit: il lavoro è un'attività conforme allo scopo che per essere tale non può non «svolgersi secondo un programma» (ivi: 16). Essendo anche l'uso-consumo basato su determinati programmi, l'uso-consumo è lavoro. $\mathrm{Al}$ contrario,

il solo modo per tenere provvisoriamente distinto l'uso dal lavoro (come il consumo dalla produzione) consiste nel dire che, dato un certo artefatto, l'uso si pone a valle di esso mentre il lavoro sta a monte. L'artefatto viene allora adoperato come pietra miliare su cui si opera la distinzione. L'uso è lavoro a valle dell'artefatto - lo è ogni volta che il discorso si limita espressamente all'immediato prima e all'immediato dopo di quel determinato artefatto. In altre parole, le qualità di essere uso e di essere lavoro si predicano simmetricamente a partire da un punto assunto come fermo nel processo che si sta descrivendo, e questo punto fermo è un artefatto. (ibidem)

Per spiegare meglio questa dinamica, Rossi-Landi evidenzia i momenti particolari che scandiscono la dialettica tra produzione ed uso: l'artefatto è un processo che si articola in «(i) modelli di produzione, (ii) esemplari di tali modelli, (iii) programmi per l'uso di esemplari, (iv) esecuzioni di tali programmi» (Rossi-Landi [1985] 2006: 19). La tesi di Rossi-Landi è che la realizzazione dell'esemplare non concluda il processo lavorativo; al contrario, il lavoro continua, assumendo la forma dell'esecuzione dei programmi d'uso degli esemplari.

Il lavoro si irraggia, distribuisce e deposita su tutto ciò in maniere ineguali; al tempo stesso, esso tiene tutto insieme. È lavoro nel senso più comunemente riconosciuto allorché si producono esemplari: dato il modello di un tavolo, un falegname ne realizza uno. Si usa ammettere che sia lavoro anche la produzione dei relativi modelli: i tavoli non esistono in natura con le loro quattro gambe, dunque ci deve essere stata la formazione storica di quel modello. Può suonare più ostico, a certe orecchie, che siano lavoro anche la produzione dei programmi d'uso e soprattutto la loro esecuzione, specie nei campi tradizionalmente riservati alla "libera attività dello spirito" o anche soltanto "dell'individuo". (ivi: 19-20)

È dunque la presenza dei programmi a porre in continuità il lavoro produttivo e l'uso-consumo: si produce seguendo programmi, si usa - e consuma 


\section{Ocula $a^{15}$}

Commemorating Charles S. Peirce (1839-1914): interpretive semiotics and mass media

Giorgio Borrelli, Consumo di merci-segni e di segni-merci nella riproduzione sociale

- eseguendo programmi.

L'Oggetto di un artefatto - abbiamo visto - è costituito dal processo di produzione e dalla funzione che l'artefatto può svolgere; più specificamente, la funzione può essere intesa come uno «schema di interazione» $\mathrm{o}$ «schema d'uso di un artefatto» (Proni 2012: 57). Le sequenze di interazione tra utente ed oggetto, previste ed attivate dallo schema d'uso, dipendono - anche - dalla forma fisica che il progettista-produttore ha dato all'artefatto, una forma studiata appositamente per supportare la funzione e da cui possono derivare tanto delle possibilità d'uso (affordance) quanto dei vincoli e dei limiti d'interpretazione (cfr. ibidem). Tali possibilità e costrizioni costituiscono le «proprietà dell'oggetto d'uso» (ivi: 58 , corsivo mio).

In modo non dissimile, Rossi-Landi sottolinea come il programma - o lo schema, per usare il termine di Proni - d'uso dipenda «dalle proprietà dell'artefatto, le quali sono state riunite e combinate nell'artefatto dal lavoro produttivo che vi ha realizzato un esemplare di un modello ben preciso» (Rossi-Landi [1985] 2006: 24-25, corsivo mio). La conclusione di Rossi-Landi è che il programma d'uso sia «connesso al modello di produzione; ma si tratta d'una connessione indiretta, perché di mezzo c'è appunto l'artefatto come totalità in sé compiuta, come risultato stabile di precedente lavoro. È per così dire solo penetrando dentro alla totalità dell'artefatto, e poi riuscendone dall'altra parte, che si opera il collegamento fra programma d'uso e modello di produzione» (ivi: 25).

"Riuscire dall'altra parte dell'artefatto" significa ritrovarsi nella dimensione dell'uso-consumo, significa scegliere di attivare o non attivare il programma d'uso inscritto nell'artefatto al momento della sua progettazione, ma significa anche dover riconoscere necessariamente di essere stati programmati in quanto utenti capaci di eseguire quel programma. Dice Rossi-Landi, se assumiamo per buono che l'uso sia lavoro "a valle" dell'artefatto - e dunque che l'artefatto sia già stato prodotto "a monte" - e che «we cannot use an artefact without at the same time following a program of use, with the aim of realizing it» (Rossi-Landi 1977: 43), allora dobbiamo riconoscere anche che «questo programma [...] in una qualche maniera [dobbiamo] pure averlo appreso, il che vuol dire che esisteva anch'esso prima di [noi]» (Rossi-Landi [1985] 2006: 24, corsivo mio). A mio modo di vedere, il fatto che un programma d'uso presupponga necessariamente una qualche forma apprendimento - $\mathrm{e}$ dunque un utente esperto, dotato di competenza (cfr. Proni 2012: 142) - è ciò che Proni ben sintetizza nel concetto di "utente implicito". L'utente implicito è «l'utente fisicamente necessario per usare l'oggetto» (Proni 2012: 58). È il processo di produzione che ipotizza l'utente implicito dell'artefatto, presupponendone determinate «abilità pratiche» (ibidem), sviluppate tramite un «addestramento specifico» (ivi: 68).

Mi sembra dunque plausibile affermare che l'approccio "peirceano" e quello di Rossi-Landi seguano la medesima traiettoria analitica: la continuità tra processo di uso-consumo e processo di produzione è ricostruibile semioticamente solo prendendo come punto di partenza il carattere di «sinapsi» (Ros- 


\section{Ocula $a^{15}$}

Commemorating Charles S. Peirce (1839-1914): interpretive semiotics and mass media

Giorgio Borrelli, Consumo di merci-segni e di segni-merci nella riproduzione sociale

si-Landi [1985] 2006: 20) dell'artefatto, ${ }^{5}$ cioè l'artefatto come punto di snodo tra il processo di produzione e quello di uso-consumo. Partendo da quest'ultimo e risalendo al lavoro produttivo che ha generato l'oggetto d'uso, potremmo dire che: a) l'uso-consumo viene a configurarsi come un'interpretazione di programmi condizionata dalle proprietà di un determinato modello (cioè, condizionata dalle opportunità e dai limiti derivanti dalla sua forma fisica); b) la forma fisica dei modelli viene progettata per consentire lo svolgimento di questi schemi d'uso, cioè per supportare una determinata funzione; c) il lavoro produttivo inscrive nei modelli di determinati artefatti degli schemi (o programmi) d'uso. Provando invece a descrivere semioticamente l'artefatto dalla prospettiva del processo di produzione, diremmo che: a) la produzione di modelli d'artefatto e di programmi d'uso presuppone un utente dotato di determinate abilità pratiche e di un determinato addestramento; e dunque, che la produzione degli artefatti e dei loro programmi viene a configurarsi come un'ipotesi interpretativa delle capacità dell'utente; b) l'artefatto funzionerà - diventando un vero oggetto d'uso (cfr. Proni 2012: 49) - se sarà in grado di interpretare e soddisfare i bisogni dell'utente; c) l'utente a propria volta dovrà essere in grado di definire i propri bisogni e di interpretarli attraverso le possibilità offerte dall'oggetto d'uso.

Utilizzando un impianto categoriale peirceano, potremmo specificare che la continuità tra i processi di produzione e uso-consumo dipende dal loro reciproco tentativo di interpretarsi, di conseguire le proprie finalità - la soddisfazione dei propri bisogni - ipotizzando determinate condizioni d'azione. Seguendo l'approccio "peirceano" di Proni, possiamo dire che i rapporti tra produzione e uso-consumo siano analizzabili rilevando determinati schemi d'inferenze, riassumibili nella forma logica «SE vuoi ottenere questo e questo, ALLORA devi fare così e così» (Proni 2012: 164). Quindi, ponendo l'uso-consumo come sistema di condizioni per la produzione e, viceversa, la produzione come sistema di condizioni per l'uso-consumo, potremmo provare a delineare le seguenti forme di inferenze: al polo della produzione avremo l'inferenza "SE l'utente sarà dotato di queste capacità, ALLORA l'artefatto potrà funzionare”; invece, al polo dell'uso-consumo avremo l'inferenza "SE voglio ottenere certi risultati, ALLORA devo utilizzare un oggetto con certe proprietà e che funzioni in questo modo" (cfr. ivi: 164).

\section{La semiosi dell'invenzione nella produzione e nel consumo}

Alla luce delle considerazioni svolte finora, mi sembra di poter affermare che la reciproca azione interpretativa di produzione e uso-consumo costituisca un'articolazione di quella che Bonfantini (1987) definisce logica elementare del processo d'azione. Secondo Bonfantini:

5 Con la metafora della "sinapsi" o della "chiusa fluviale" Rossi-Landi vuole mostrare come il lavoro - inteso come zweckmässige Tätigkeit - "inizi” con la produzione dell'artefatto, si "fermi" momentaneamente in esso (come l'acqua di un fiume in una chiusa, o come un impulso nervoso in una sinapsi), e poi "prosegua" nuovamente nel processo di uso-consumo di quell'artefatto (cfr. Rossi-Landi [1985] 2006: 20) 


\section{Ocula $a^{15}$}

Commemorating Charles S. Peirce (1839-1914): interpretive semiotics and mass media

Giorgio Borrelli, Consumo di merci-segni e di segni-merci nella riproduzione sociale

L'agire umano sorge come risultato di una contraddizione fra 1) il soggetto umano come soggetto ricco di bisogni intenzionalmente disposti e 2) una determinata situazione oggettiva che si oppone ai bisogni e li fa scattare.

Questa prima contraddizione porta alla identificazione della situazione oggettiva come problema - primo risultato -, o interpretante della prima interpretazione o interpretazione fondativa di un ciclo di interpretazioni. Di qui il porsi di una seconda contraddizione fra 1) il bisogno come già orientato in desiderio ovvero desiderio toutcourt e 2) il problema.

Questa seconda contraddizione porta a una sua interpretazione, o meglio è interdipendente rispetto alla sua interpretazione: l'identificazione o individuazione di un'area di soluzione come orizzonte teleologico (area delle prospettive di valore).

La contraddizione fra il desiderio e l'orizzonte teleologico porta allo stabilirsi del valore o fine.

Il rapporto fra desiderio e fine porta alla riflessione sui mezzi. (Bonfantini 1987: 35)

Il processo di azione è attivato da bisogni intenzionalmente disposti, ovvero da bisogni già interpretati come conseguenze di una determinata situazione oggettiva. A seguito di questa interpretazione fondativa, la relazione causale tra situazione oggettiva e bisogno assume la forma del problema; l'ipotesi di soluzione del problema costituirà la sua interpretazione, divenendo lo scopo verso cui è orientato l'agire. L'individuazione dei mezzi opportuni per la soluzione del problema costituirà il programma (cfr. ibidem). Secondo Bonfantini, questo processo può essere scandito da tre momenti, ciascuno dei quali rappresenta un atto semiotico interpretativo: a) la rappresentazione (movimento dall'evento al segno); b) la proiezione (movimento dal segno e dal desiderio alla meta); c) la programmazione (movimento dal segno e dalla meta al progetto). «Il passaggio da un atto semiosico compiuto a un nuovo atto semiosico è determinato dal soddisfacimento della prima intenzione, o dall'irruzione e percezione di nuovi eventi divergenti, o dall'insorgere di una qualunque resistenza alla soddisfazione» (ibidem).

L'agire dunque prende avvio dall'interpretazione dei bisogni mediante i desideri e prosegue con l'elaborazione di questi ultimi in problemi; i desideri - strutturati in problemi - vengono a loro volta elaborati in finalità, scopi da conseguire e che orientano l'azione. L'azione si conclude con la progettazione dei mezzi per appagare quei desideri (soddisfacendo il bisogno iniziale).

A partire da questa logica elementare, Bonfantini delinea un' «interpretazione pragmaticista del materialismo storico» (ivi: 91). Prendendo le distanze dalla concezione malinowskiana dei bisogni come «fondamento biologico della cultura» (Rossi-Landi [1985] 2006: 232) e dalle letture "marxiste ortodosse", che intendono il desiderio come mera «manifestazione del bisogno» (Bonfantini 1987: 158), Bonfantini propone di considerare i desideri come «interpretazioni di un rapporto problematico bisogno-ambiente in vista del soddisfacimento» (ibidem). La radice teorica di questa rilettura è chiaramente individuabile (v. Proni 2007: 95) in quanto Peirce afferma a proposito dell'osservazione astrattiva, e cioè che 


\section{Ocula $a^{15}$}

Commemorating Charles S. Peirce (1839-1914): interpretive semiotics and mass media

Giorgio Borrelli, Consumo di merci-segni e di segni-merci nella riproduzione sociale

È esperienza familiare a ogni essere umano desiderare qualcosa molto al di là dei propri mezzi attuali e far seguire a questo desiderio la domanda «Desidererei quella cosa con la stessa intensità, se avessi a disposizione i mezzi per procurarmela?» Per rispondere questa domanda egli indaga nel proprio animo, e così facendo opera ciò che io chiamo osservazione astrattiva. Costruisce nella sua immaginazione un diagramma, o schema sommario rappresentativo, di se stesso; considera quali modificazioni lo stato ipotetico delle cose richiederebbe che venissero fatte in questo quadro, e poi le esamina, cioè osserva ciò che ha immaginato per vedere se vi si può distinguere lo stesso ardente desiderio. (CP 2.227 2003: 147)

L'osservazione astrattiva è «il processo interno, naturale e spontaneo, attraverso il quale un soggetto costruisce un modello semiotico [... ] che parte dalla situazione di fatto [...], e la manipola [...]. Si tratta di costruire, secondo la teoria pragmatica, le conseguenze pratiche di una situazione» (Proni 2012: 95, corsivo mio). Nel caso esemplare considerato da Peirce, si tratta di immaginare la propria capacità di «soddisfare un desiderio che al momento è impossibile» (ibidem). La costruzione del modello semiotico coincide con «lo schema del progetto» (ivi: 96) e ha carattere abduttivo, dato che il progetto "procede dall'effetto alla causa» (ivi: 95). Più specificamente, il progetto rappresenta «un obiettivo dal quale abdurre le azioni da intraprendere per raggiungerlo [...]. Il soggetto costruisce un interpretante dell'obiettivo che intende raggiungere, di sé stesso e dei cambiamenti che dovrebbe attuare per raggiungerlo. Confronta in sostanza il proprio fine con uno scenario e con l'azione per realizzarlo. E questo è il senso fondamentale del progetto» (ivi: 96).

Dunque, a partire dal materialismo pragmaticista di Bonfantini, si può sottolineare come una semiotica del processo di produzione e consumo non possa prescindere dall'analisi dei progetti attraverso cui i soggetti elaborano i propri desideri e problemi. Alla luce di questa considerazione, mi sembra di poter individuare un punto d'incontro tra la semiotica di Bonfantini e quella di Rossi-Landi: il bisogno non può essere analizzato se non come elemento interno ai processi interpretativi che articolano la produzione e l'uso-consumo. Ciò non vuol dire negare che i bisogni non costituiscano «la condizione materiale scatenante il desiderio» (Bonfantini 1987: 158), ma vuol dire negare che essi siano entità "naturali" che svolgono un ruolo determinante nel processo di riproduzione sociale. Ritengo che Rossi-Landi si attesti sulle medesime posizioni, e ciò si può evincere da alcune sue considerazioni sull'"Einleitung" del 1857, lo scritto in cui Marx capovolge l'idea che i bisogni costituiscano la base "naturale" della cultura, affermando, al contrario, che essi prendano forma «allinterno del processo di produzione e consumo» (Rossi-Landi [1985] 2006: 232).

Secondo Marx - abbiamo visto all'inizio -, tra produzione e consumo si svolge un

movimento di mediazione. La produzione media il consumo, di cui crea il materiale e al quale senza di essa mancherebbe l'oggetto. Ma il consumo media a sua volta la produzione, in quanto solo esso procura ai prodotti il soggetto per il quale essi sono dei prodotti. Il prodotto riceve il suo ultimo finish [perfezionamento] soltanto nel consu- 


\section{Pcula $a^{15}$}

Commemorating Charles S. Peirce (1839-1914): interpretive semiotics and mass media

Giorgio Borrelli, Consumo di merci-segni e di segni-merci nella riproduzione sociale

mo. Una ferrovia sulla quale non si viaggi e che quindi non si logori e non venga con-

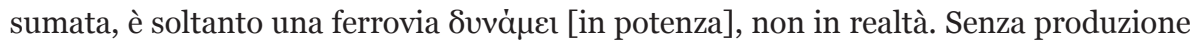
non v'è consumo; ma non v'è nemmeno una produzione senza consumo, altrimenti la produzione sarebbe senza scopo. (Marx 1968: tr. it. 15)

Dunque, già in Marx è presente l'idea che l'artefatto sia effettivamente un artefatto solo nel caso in cui la sua funzione possa essere svolta (cfr. Proni 2012: 49); in caso contrario, sarebbe un artefatto (ad esempio, una ferrovia)

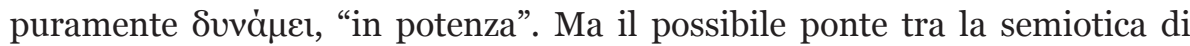
Bonfantini e quella di Rossi-Landi si può ritrovare - a mio parere - in quanto sto per dire: Marx, nel trarre le sue conclusioni a proposito della dialettica tra produzione e consumo, parte proprio dall'artefatto, intendendolo come il risultato di un processo in cui la produzione dà ai bisogni la forma degli oggetti attraverso cui i bisogni stessi possono essere soddisfatti, definendo inoltre il modo in cui soddisfare i bisogni attraverso l'uso degli oggetti.

Insomma - dice Marx -, l'oggetto non è un oggetto in generale, ma un oggetto determinato, che deve essere consumato in un modo determinato, in un modo che a sua volta dev'essere mediato dalla produzione stessa. La fame è fame, ma la fame che si soddisfa con carne cotta, mangiata con coltello e forchetta, è una fame diversa da quella che divora carne cruda, aiutandosi con mani, unghie e denti. Non è soltanto l'oggetto del consumo dunque ad essere prodotto della produzione, ma anche il modo di consumarlo, non solo oggettivamente, ma anche soggettivamente. La produzione crea quindi il consumatore. (Marx 1968: tr. it. 16)

In questa prospettiva, continua Marx, la propensione al consumo è necessariamente mediata dall'oggetto; ciò vuol dire che il bisogno che si avverte dell'oggetto «è creato dalla percezione dell'oggetto stesso» (ibidem). In sintesi,

La produzione produce [...] il consumo 1) creandogli il materiale; 2) determinando il modo di consumo; 3) producendo come bisogno nel consumatore i prodotti che essa ha originariamente creato come oggetti. Essa produce perciò l'oggetto del consumo, il modo di consumo. Allo stesso modo il consumo produce la disposizione del produttore, sollecitandolo in veste di bisogno che dà una finalità alla produzione. (ivi: 17)

In modo più preciso, il consumo «crea il bisogno di una nuova produzione e quindi quel motivo ideale che è lo stimolo interno della produzione e il suo presupposto. Il consumo crea la propensione alla produzione; esso crea anche l'oggetto, che determina finalisticamente la produzione, come bisogno, come propensione e come scopo» (ivi: 15-16).

Seguendo l'impostazione teorica di Bonfantini e Proni - dunque, leggendo la dialettica tra questi due momenti economici attraverso la logica elementare del processo di azione e l'osservazione astrattiva - potremmo dire che produzione e uso-consumo si pongono reciprocamente "in veste di bisogni", cioè sollecitano la reciproca elaborazione dei bisogni intesi come conseguenze di una situazione oggettiva problematica che necessita una risposta. La produ- 


\section{Ocula $a^{15}$}

Commemorating Charles S. Peirce (1839-1914): interpretive semiotics and mass media

Giorgio Borrelli, Consumo di merci-segni e di segni-merci nella riproduzione sociale

zione interpreta un determinato bisogno derivante dalla sfera del consumo e lo pianifica, progetta il modo di soddisfarlo; nel pianificare dà forma a quel bisogno, lo struttura come desiderio. In questo senso, il consumo è un elemento interno alla processualità della produzione, perché costituisce l'elemento da cui la produzione prende avvio e che la produzione elabora. In sintesi, dire con Marx - che «la produzione fornisce non solo un materiale al bisogno, ma anche un bisogno al materiale» (ivi: 16) significa dire - a mio modo di vedere - che i bisogni di uso-consumo costituiscano l'Oggetto della produzione, che essa inscriva negli artefatti degli interpretanti finali, degli «schemi di risposta» (Proni 2012: 96) per quei bisogni.

Al polo opposto, l'uso-consumo dispone i propri interpretanti finali come risposte al bisogno della produzione di trovare quel momento economico che le darà la sua determinatezza, realizzandone gli scopi. L'uso-consumo struttura i suoi desideri a partire dalle condizioni oggettive poste dalla produzione, cioè dal modo in cui la produzione ha a propria volta già interpretato e inscritto i bisogni dell'uso-consumo negli artefatti. La produzione è, in questa prospettiva, il momento egemonico, cioè «il punto di partenza della realizzazione, l'atto nel quale l'intero processo riprende il suo andamento» (Marx 1968: tr. it. 19).

\section{Conclusione. Verso una semiotica dei programmi di scambio}

Il movimento di mediazione tra produzione e uso-consumo appare lineare, ma non lo è. Non lo è per un motivo elementare: il consumo è solo uno dei fini della produzione. Il soddisfacimento dei bisogni del consumatore nelle società caratterizzate da un'economia capitalistica è mediato - per non dire fortemente condizionato? - dalle esigenze di valorizzazione del capitale. Per dirla con Marx, tra il momento della produzione e quello dell'uso consumo «s'interpone la distribuzione» (ibidem), più specificamente, il mercato come dimensione della distribuzione nelle società capitalistiche.

Il fatto che la produzione capitalistica sia produzione per il mercato implica che l'interpretazione dei bisogni derivanti dalla sfera del consumo non sia l'unico fattore ad attivare la progettualità della produzione. Questo punto è ribadito da Bonfantini, che sottolinea come l'analisi semiotica non debba tralasciare il ruolo giocato «dal mercato e dall'imprenditoria» (Bonfantini 2004: 13) nel cammino dell'invenzione progettuale. In sostanza - e questo mi sembra un ulteriore elemento di convergenza tra l'approccio materialistico-pragmaticista e quello rossilandiano - una semiotica che voglia analizzare la continuità tra i comportamenti di consumo e gli schemi d'utilizzo inscritti negli artefatti deve assumere che la produzione non sia semplicemente produzione di oggetti d'uso, ma sia - specificamente - produzione di merci o, come dice Rossi-Landi, produzione di messaggi-merci.

Ogni merce - fa notare Rossi-Landi - si presenta con dentro dei significati [...] Una merce non va al mercato da sola: ha bisogno di un venditore; e non viene venduta fino a che non ci sia qualcuno che la compera, cioè l'accetta in scambio con denaro, ovvero con altre merci se siamo a livello del baratto. Inol- 


\section{Ocula $a^{15}$}

Commemorating Charles S. Peirce (1839-1914): interpretive semiotics and mass media

Giorgio Borrelli, Consumo di merci-segni e di segni-merci nella riproduzione sociale

tre un prodotto non si trasforma in merce come un bruco in farfalla, bensì solo in quanto gli uomini lo immettano in determinati rapporti significanti; né una merce può venir adoperata come prodotto (anzi come mero bene), se merce era, senza che con ciò si prescinda dal suo esser stata merce. Insomma, [...] una merce è merce anziché prodotto in quanto è messaggio. ${ }^{6}$ (Rossi-Landi [1968] 2003: 108-109)

In questa prospettiva, la semiotica dovrebbe includere nell'ambito d'analisi lo studio dei programmi «che entrano in gioco affinché fra la produzione e il consumo si inserisca [...] lo scambio» (Rossi-Landi 1985 2006: 34). L'individuazione e la descrizione di questi programmi - nonché delle ulteriori progettazioni da essi derivanti - sono compiti che esulano da questo lavoro. Tuttavia, ritengo di poter affermare che delle traiettorie di ricerca siano presenti in nuce nella problematica dell'obsolescenza programmata degli artefatti: il fatto che un oggetto d'uso abbia un ciclo di vita progettato in fase di produzione, e che tale obsolescenza sia funzionale al profitto potrebbe spingerci ad ipotizzare che vi sia un continuum non solo tra la progettazione degli artefatti e la loro funzione pratica, ma anche tra la progettazione degli artefatti ed il processo di valorizzazione del capitale. Del resto, sottolinea Proni, «il sistema del consumo si è sviluppato incentivando l'obsolescenza dei prodotti per aumentare la frequenza di sostituzione, e finché il potere d'acquisto e la propensione al consumo restano alti il processo procede abbastanza fluidamente. Usiamo prodotti opachi finché giudichiamo che siano obsoleti (a volte solo esteticamente) e poi li facciamo riparare da esperti o ne acquistiamo altri, possibilmente ritenuti migliori (più evoluti, più belli, più efficienti, ecc.)» (Proni 2012: 40). Ponzio (2013: 27) ha mostrato come l'obsolescenza programmata rientri addirittura nelle strategie economiche di "innovazione" della Commissione Europea.

In questa prospettiva, forse, non sarebbe poi così sorpassato un approccio marxista alla teoria di bisogni (v. Heller 1974) dal momento che - mi sembra di poter affermare - continuiamo a vivere «in una società in cui il fine della produzione non è la soddisfazione di bisogni, ma la valorizzazione del capitale, in cui il sistema dei bisogni è fondato dalla divisione del lavoro e il bisogno compare soltanto sul mercato, nella forma di domanda solvibile» (Heller 1974: 26).

6 Secondo Ponzio, nella fase odierna dello sviluppo capitalistico - detta della comunicazione-produzione - «la comunicazione si presenta come fattore costitutivo della produzione» (Ponzio 2008: 14). La produzione è produzione di messaggi, scambiati sul mercato come se fossero merci, e il consumo è fondamentalmente consumo di comunicazione. In questa prospettiva «è ormai abbastanza evidente che non solo le merci sono messaggi, ma anche che i messaggi sono merci. L'intera produzione è comunicazione e viceversa» (ibidem). 
Giorgio Borrelli, Consumo di merci-segni e di segni-merci nella riproduzione sociale

\section{Bibliografia}

Bonfantini, Massimo A.

1987 La semiosi e l'abduzione, Milano, Bompiani.

2003 (a cura di), Opere, di Peirce, C.S., con la collaborazione di G. Proni, Milano, Bompiani.

2004 "La semiosi dell'invenzione progettuale", in Bonfantini, M.A. e Terenzi, M. (a cura di), Come inventare e progettare alla maniera di Poe. Filosofia della composizione, Bergamo, Moretti-Honegger.

Heller, Ágnes

1974 La teoria dei bisogni in Marx, Milano, Feltrinelli.

Marx, Karl

1968 Lineamenti fondamentali della critica dell'economia politica 1857-1858. Volume I, Torino, Einaudi.

Peirce, Charles Sanders

2003 Opere, Bonfantini, M.A. (a cura di), con la collaborazione di G. Proni, Milano, Bompiani.

Petrilli, Susan

1999 "La biosemiotica di Morris", in Petrilli, S. (a cura di), Lineamenti di una teoria dei segni, di Morris, C., Lecce, Piero Manni.

2004 "Corpi e segni. Per una tipologia della materialità semiosica”, Athanor. Lavoro immateriale, 7, 2003-2004, pp. 151-165.

Ponzio, Augusto

2004 Semiotica e dialettica, Bari, Edizioni dal Sud.

2008 Linguaggio, lavoro e mercato globale. Rileggendo Rossi-Landi, Milano-Udine, Mimesis

2012 Ferruccio Rossi-Landi e la filosofia del linguaggio, Lecce, Pensa MultiMedia.

2013 Fuori luogo. L'esorbitante nella riproduzione dell'identico, Milano-Udine, Mimesis.

Proni, Giampaolo

1990 Introduzione a Peirce, Milano, Bompiani.

2012 La lista della spesa e altri progetti. Semiotica, design e comportamenti delle persone, Milano, Franco Angeli.

Rossi-Landi, Ferruccio

1968 Il linguaggio come lavoro e come mercato. Una teoria della produzione e dell'alienazione linguistiche, Milano, Bompiani, 20035.

1972 Semiotica e ideologia, Milano, Bompiani, 20115.

1975 Charles Morris e la semiotica novecentesca, Milano, Feltrinelli.

1977 Linguistics and economics, L’Aia-Parigi, Mouton.

1978 Ideologia, Roma, Meltemi, 20053.

1985 Metodica filosofica e scienza dei segni, Milano, Bompiani, $2006^{2}$.

Stern, Robert

2009 Hegelian Metaphysics, Oxford, Oxford University Press.

Giorgio Borrelli è laureato in "Sociologia e Ricerca Sociale" presso l'Università degli Studi di Trento. È attualmente dottorando in "Teoria del linguaggio e scienze dei segni” presso l’Università degli Studi di Bari “Aldo Moro”. Il suo progetto di dottorato è incentrato sulla semiotica materialistica di Ferruccio Rossi-Landi. 